\title{
The influence of endogenous surfactant on the structure and drug-release properties of Eudragit NE30D-matrices
}

\author{
Achim Göpferich and Geoffrey Lee \\ Institute for Pharmaceutical Technology and Biopharmaceutics, Heidelberg University, Heidelberg, Germany
}

(Received 22 February 1991; accepted in revised form 22 August 1991)

The influence of an endogenous surfactant present in Eudragit NE3OD on the structure and drug release (clenibuterol) properties of thin matrices has been examined. Both drug-free and drug-loaded matrices were found to be non-isotropic in structure, the former having a marbled appearance under the polarising light microscope, and the latter showing numerous needle-shaped crystals. At loading above approx. $10 \% \mathrm{w} / \mathrm{w}$ clenbuterol it was also possible to observe aggregates of the drug. Differential scanning calorimetry enabled the identification of melting peaks at approx. $50^{\circ} \mathrm{C}$ for the weedle-shaped crystals and approx. $80^{\circ} \mathrm{C}$ for the larger drug aggregates. The former are composed of a surfactant used by the manufacturer for the synthesis of Eudragit NE30D by emulsion polymerization. This surfactant undergoes a phase separation from the polymer on storage at room temperature. It could, however, be extracted from the polymer by refluxing in water to yield an isotropic system. The extract showed a melting peak at $50^{\circ} \mathrm{C}$ and also UV, IR, NMR, and mass spectra in accordance with an o-substituted nonyl phenol surfactant. Matrices prepared from the purified Eudragit NE30D showed drug release rates of only one third the magnitude of those found with matrices prepared from the raw polymer. Substantially reduced scatter in the release data was also found with the purified polymer.

Key words: Pulyatrylate polymer; Surfactant; Diffusion

\section{Introduction}

We have been interested for some time in the use of Eudragit NE30D as a carricr for the sustained release of drugs. Matrix-type carriers can easily be prepared from thin films of the polymer cast by solvent evaporation [1]. The rate of release of the drug clenbuterol from such matrices has been examined using a simple diffusion cell [2]. The accurate evaluation of the kinetic data so obtained requires, however, knowledge of the

Correspondence to: G. Lec, Institute for Pharmaceuticol Technology and Biopharmaceutics, Heidelberg University, Im Neuenheimer Feld 366, D6900 Heidelberg, Germany. mechanism of drug release from the polymer. of eiemental importance in this respect is the physical state of the drug within the polymer matrix, as the release kinetics depend inter alia on whether the drug exists in the dissolved or suspended condition [3]. We deemed it expedient, therefore, to determine the solubility of the clenbuterol in the Eudragit NE30D. To that end an examination of drug-loaded polymer matrices was undertaken using polarising light microscopy. We were surprised to observe that the matrices were strikingly anisotropic in structure, showing either a marbled appearance or the presence of variously-shaped crystals and fractal aggregates. Indeed, it was not possible to distin- 
guist: unequivocally between drug crystals and the other structures observed. We found litile information in the literature concerning the structure of Eudragit NE30D, despite its use to produce matrix tablets as a drug carrier [4]. Only two differential scanning calorimetric studies of Eudragit NE30D [5] and the related Eudragit 7972-55 PMMA ON [6] have been published, neither of which offered a satisfactory explanation of our observations.

It soon became clear from further experiment. that the puzzling microscopical appearance of the matrices was a result of the presence of small amounts of a water-soluble contaminant within the Eudragit NE3OD. This is almost certainly a surfactant used by the manufacturer for the synthesis of the polymer by emulsion polymerization [ $7 \mathrm{a}$ ]. It was possible to remove this endogenous contaminant from the polymer by extraction with water to yield an isoiropic system. We present in this paper our findings concerning the effects of the endogenous surfactant on the physical state of clcibuterol within the Eudragit NE30D-matrices and the consequences for drug release rate which ensue from its removal.

\section{Materials and Methods}

\section{Materials}

The basic drug clenbuterol of molecular weight 277 and pKa 9.5 was used as received from Boehringer Ingelheim $\mathrm{KG}$ (Ingelheim, Germany):

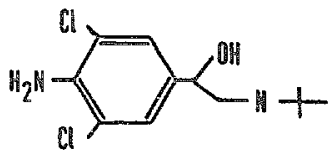

Eudragit NE30D (Röhm, Weiterstadt, Germany) is a neutral, poly (ethylacrylate-methylmethacrylate) copolymer with a molecular weight of approx. 800,000 , and which exists in the rubbery state at room temperature (glass transition at $-8^{\circ} \mathrm{C}$ ):

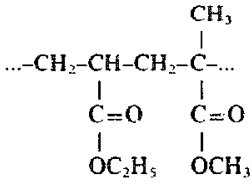

Although insoluble at all pHs, it swells in water. It was obtained as a $30 \%$ aqueous dispersion and freeze dried at $-25^{\circ} \mathrm{C}$ to yield a white solid. All solvents and buffer salts were pA grade. Water was double-distilled from an all-glass apparatus.

\section{Experimental methods}

Preparation of Eudragit NE30D-matrices

Films of $50 \mu \mathrm{m}$ thickness containing various concentrations of clenbuterol in the range 0-20\% $w / w$ were prepared on a siliconized paper base by the casting of a $15 \% \mathrm{w} / \mathrm{w}$ acetone sulution of the raw polymer. The product was dried at room temperature for $12 \mathrm{~h}$, after which less than 50 ppm acetone remained as determined by gas chromatography. Round matrices $(\varnothing 2 \mathrm{~cm})$ were then cut from the film using a punch.

According to the manufacturer, Eudragit NE30D contains traces of a water soluble, neutral surfactant of the nonyl-phenol-type [7b]. We attempted to extract the surfactant from Eudragit NE30D by refluxing the freeze-dried polymer in a Soxlet for $90 \mathrm{~h}$ with water. The resulting purified polymer was a white solid. The dried extract took the form of a yellowish-white, crystalline solid, which was found to represent approx. $3.3 \%$ of the total mass of the dried raw polymer. Matrices of the purified Eudragit NE30D were then prepared as described abovs, also containing concentrations of clenbuterol in the range 0 $20 \% \mathrm{w} / \mathrm{w}$.

\section{Polarising light microscopy}

The matrices were examined at various times after their preparation using a Zeiss Standard microscope fitted with a polarizing objective and condenser, a hot stage for examining thermal behaviour, and a Nikon C35 camera. The extract and pure clenbuterol were also examined. 
Diflerential scanming calorimetry (DSC)

A Mettler model TC10A (Greifensee, Switzeriand) was used to examine the matrices at various times after their preparation, as well as the exiract and pure clenbuterol. $A$ heating/cooling speed of $10 \mathrm{~K} / \mathrm{min}$ was selected.

\section{UV, lR and mass spectrophotometry; NM嫼}

An attempt was made to confirm if the crystalline extract obtained from the raw polymer con-
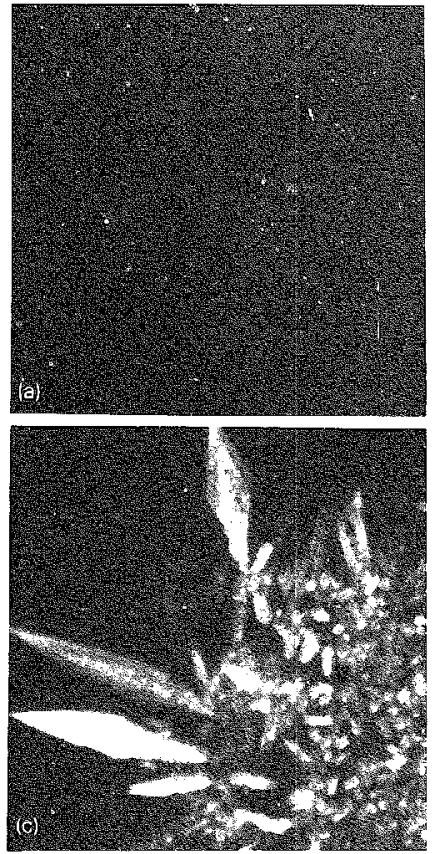

tained a surfactant of the type declared by the manufacturer. To that end, UV (Zeiss DMR 21), IR (Beckman Aculab 6), and mass spectrophotometry were employed. $250 \mathrm{MHz}$-NMR spectra were also obtained in $\mathrm{D}_{2} \mathrm{O}$. In all cases the solid extract was firsi recrystallized from methanol to remove any inorganic contaminants.

\section{Mhasurement of drug release}

The release of clenbuterol out of matrices prepared from both raw and purified Eudragit
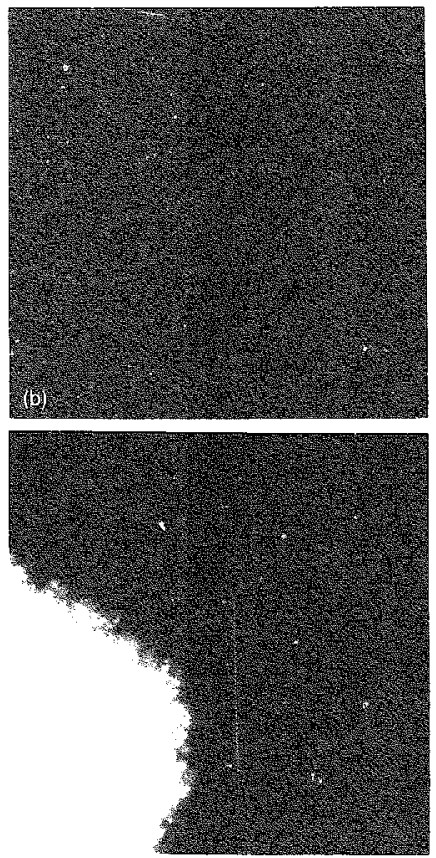

Fig. I. Photographs of 8-days' old matrices prepared from raw Eudragit NE30D viewed under crossed polarisers; (a) drug-free, (b) $6 \% \mathrm{w} / \mathrm{w}$ drug (clenbuterol) loading, (c) $12 \%$ drug loading. (d) $20 \%$ drug loading. 
NE30D into pH 8 phosphate buffer was measured at $35^{\circ} \mathrm{C}$ using an all-glass diffusion cell of standard design [2]. The results were expressed as release profiles of $m(t) / A$ versus time, where $m(t)$ is the mass of clenbuterol in the acceptor medium at time $t$ and $A$ is the release area. The diffusivity of clenbuterol in the matrices was then calculated by fitting each release profile to a numerical solution to the diffusion equation under non-sink conditions, as described in detail elsewhere [2]. Or relevance to this study is that the solution assumes the linear diffusion of drug with

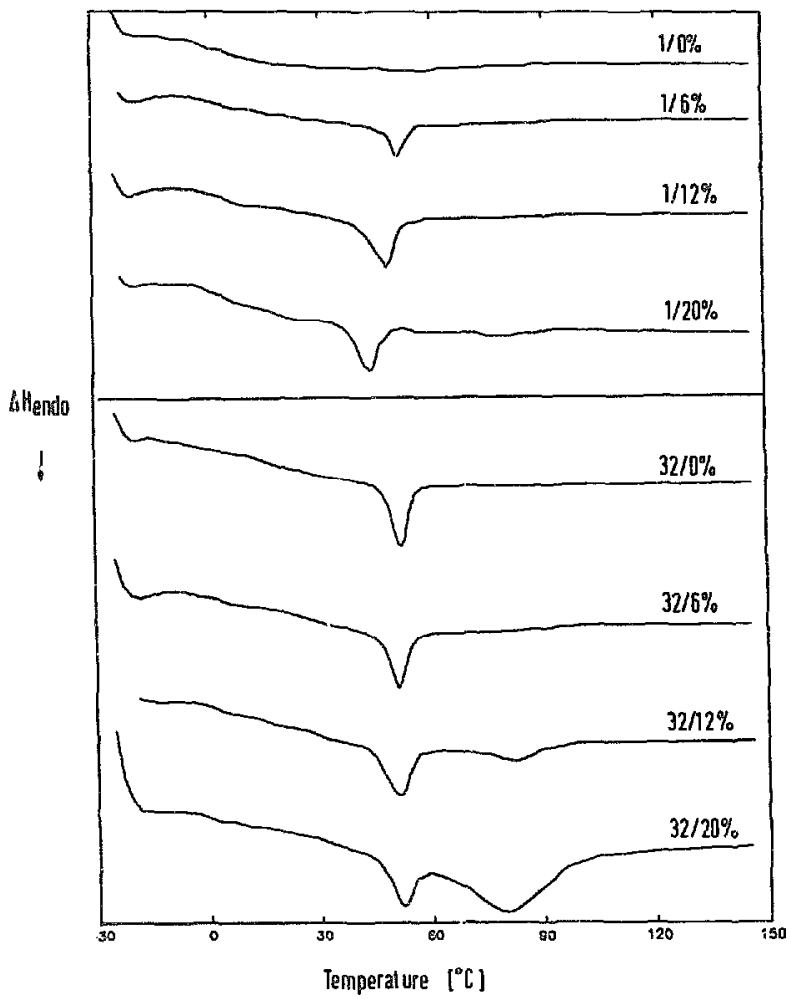

Fig. 2. DSC-scans of elenbuterol-ioaded. raw Eudragit NE30D matrices taken I and 32 days after their preparation. Key: day/ drug loading [\%]. 
constant diffusivity, D, in a finite, isotropic, plane matrix with spontaneous partitioning at all boundaries. The initial condition used specifies the existence only of dissolved drug.

\section{Fesults and Discussion}

\section{Strutture of Eudragit NE30D-matrices}

When viewed under the light microscope with crossed polarizers the raw Eudragit NE30D-matrices reveal a complex structure that depends on time elapsed since preparation and drug-loating. A stable picture first emerges after $6-8$ days" storage at room temperature. This is illustrated by the photographs taken of 8-days' old Eudragit NE30D-matrices having selected loadings of $0 \%$, $6 \%, 12 \%$, and $20 \%$ w/w clenbuterol (Fig. lam, respectively). Thus, the two matrices of highest drug loading clearly show a population of large, irregularly-shaped crystals, some of which are not aclidian but rather of fractal shape. These were observed to melt when the temperature was raised to approx. $80-90^{\circ} \mathrm{C}$, and certainly come from the incorporated drug, even though this melting point lies well below that of pure clenbuterol $\left(135^{\circ} \mathrm{C}\right)$. Careful scrutinization of these two photographs also reveals the presence of a second type of much smaller, needle-shaped crystal, which melted at approx. $50^{\circ} \mathrm{C}$. The matrix containing only $6 \% \mathrm{w} / \mathrm{w}$ clenbuterol (Fig. 16) shows none of the large crystals or fractal forms, indicating that the drug is soluble in the polymer at this concentration. The needle-shaped crystals are, however, still clearly visible at this drug loading. Although they do not appear within the drug-free matrix (Fig. Ia), the polymer has a distinctly marbled appearance under crossed polarisers and is thus also anisotropic.

The DSC-scans for these matrices (Fig. 2) at $12 \%$ and $20 \%$ drug loating both develop on storage a slight endothermic transition at approx. $80^{\circ} \mathrm{C}$. This coincides with the meling of the large crystals and fractal aggregates of drug within the polymer at this temperature, as could be ob- served directly under the microscope. Accordingly, this peak is not seen on the scans for matrix containing $6 \%$ clenbuterol, where the microscopy resulis indicate that the drug is fully dissolved, or the drug-free matrix. With all the natrices examined, bowever, a cleas endothermic peak is visible at approx. $50^{\circ} \mathrm{C}$. For the drug free matrix, this peak develops slowly on storage, first appearing clearly after 8 days. Simultaneously the marbled areas become noticeable within this matrix. All of the drug-containing matrices show a clear peak even after the first day's storage. The needle-shaped crystals can be observed to grow correspondingly. This endothermic peak is, therefore, clearly not caused purely by the existence of drug within the polymer, developing even for the drug-free matrix, albeit more slowly.

The existence of such an endothermic peak has already been noted in the literature [6] and assigned to a glass transition of the polymer at this temperature. Furthermore, there exists a proposal [5], also based on the results of DSC measurements, that crystalline structure exists within Eudragit NE30D. It is not, however, possible to reconcile the results of our experiments with this earlier work. Thus, the DSC-scan for the drugfree matrix prepared from the purifed Eudragit NE30D shows no signs of an endothermic transition at $50^{\circ} \mathrm{C}$, even after storage at room temperature for 128 days (Fig. 3 ). The thermogram for the crystalline solid extracted from the Eudragit NE30D (Fig. 4), however, clearly shows such a peak at $50^{\circ} \mathrm{C}$, corresponding to the melting point of this extract as observed under the light microscope. When a drug-free matrix prepared from the purified Eudragit NE3OD is viewed under the light microscope an isotropic system is seen (Fig. 5a); the marbled areas ob served within the raw polymer are not present and do not develop on storage. Furthermore, the matrix prepared from the purifed Eudragit NE30D and containing $6 \%$ clenbuterol (Fig. $5 \mathrm{~b}$ ) shows neither drug nor needle-shaped crystals and its DSC-scan has no visible peaks (Fig 3). With 12 and $20 \%$ drug loading the usual crystal and fractal forms of the drug can be observed un* 


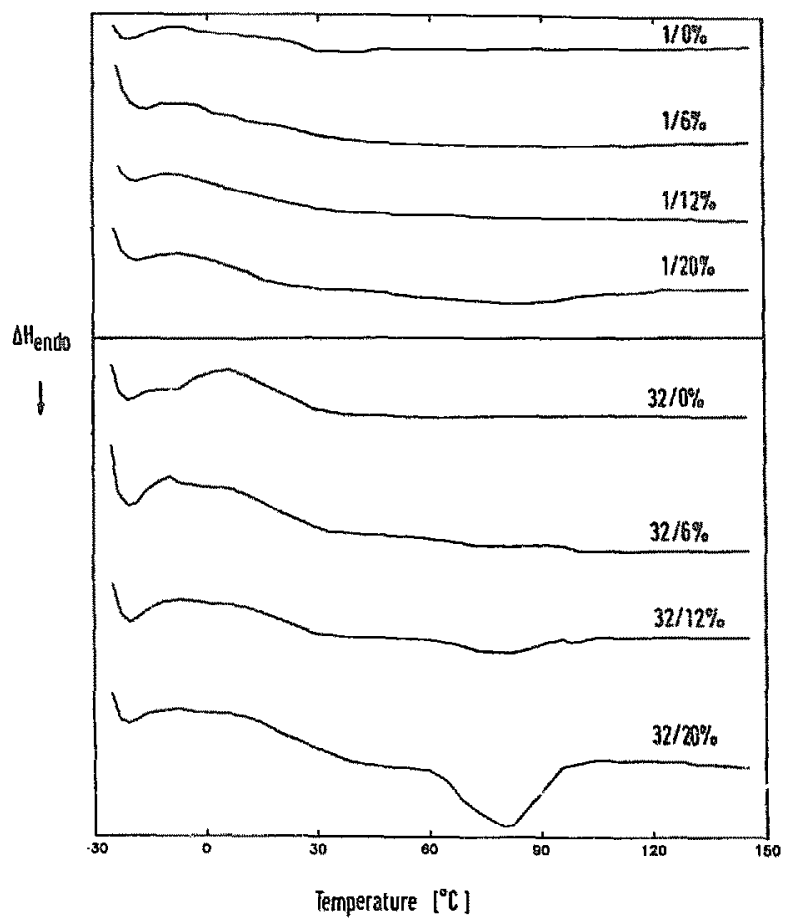

Fig. 3. DSC-seans of cienbuterol-londed, purfied Eudragit NE3OD matrices taken I and 32 days after their preparation. Key: day/drug loading [\%].

der the microscope (Fig. $5 c_{y} \mathrm{~d}$ ), but again none of the needle-shaped crystals. The DSC-scans show only the slight melting peaks for the drug crystals and aggregates at $80-90^{\circ} \mathrm{C}$ and no endothermic transition at $50^{\circ} \mathrm{C}$ (Fig. 3 ).

On the basis of these results it appears that the endothermic transition observed at approx. $50^{\circ} \mathrm{C}$ for the raw Eudragit NE30D arises from the phase separation of some water-soluble, endoge- nous substance within the polyrner. At this point we must bear in mind the manufacturer's declaration that Eudragit NE30D contains traees of a water-soluble, $\theta$-substituted nonyl-phenol surfactant having a molecular weight of 5000-6000 [7b]. The spectroscopic data we obtained from the extract provides evidence to suggest that the extract contains such a surfactant. Thus, the strong UV-absorbance of the extract at $247 \mathrm{~nm}$ 


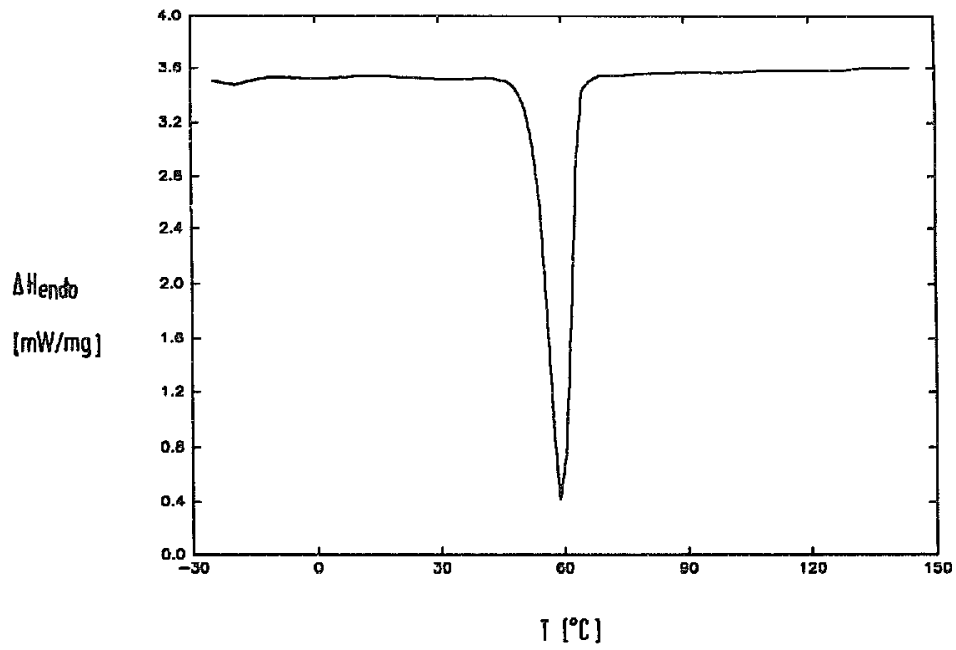

Fig. 4. DSC-scan of crystalline solid extracted from raw Eudragit NE30D.

(Fig. 6a) indicates a substituted aromatic structure. The band seen in the IR spectrum (Fig. 6b) at $2900 \mathrm{~cm}^{-1}$ comes from C.H vibrations, whilst that at $830 \mathrm{~cm}^{-1}$ can be assigned to out-of-plañe vibrations of an $\sigma$-substituted aromatic. Furthermore, the large band at $1100 \mathrm{~cm}^{-1}$ indicates a large content of $\mathrm{C}-\mathrm{O}$ groups, as would arise from a high degree of ethoxylation of the molecule. The three major peaks of the mass spectrum (Fig. $6 \mathrm{c}$ ) occur at $45 \mathrm{~m} / \mathrm{e}, 89 \mathrm{~m} / \mathrm{e}$ and $133 \mathrm{~m} / \mathrm{e}$, the difference between each being always 44 units. This finding can be explained if ethoxy chains are broken up into units of $\left[-\mathrm{CH}_{2}-\mathrm{O}-\mathrm{C}_{2} \mathrm{H}_{2}-\right]$. The NMR spectrum (Fig. 6d) can be used to estimate the ratio between aromatic protons (peaks at 6.8 and $7.2 \mathrm{ppm}$ ) and protons originating from the supposed ethoxy groups (peak at $3.7 \mathrm{ppm}$ ). $A$ value of approx. $100-150$ ethoxy units is obtained. Although these spectra are not sufficient per se to allow an unequivocal identification of the substance, it seems very probable. Any incompatibility of this substance with the polyacrylate - by virtue of the former's hydrophilicity - would lead to a phase separation from the polymer on storage. In a drug-free matrix this acsults in the marbled appearance of the polymer illustrated in Fig. 1a. The presence of drug within the matrix evidently causes the surfactant to crystallize out rapidly as needle-shaped crystals (cf. Fig. 1b).

\section{Drug release from Eudragit NE30D-mastrices}

The extraction of the endogenous contaminant from Eudragit NE30D affects the rate of release of clenbuterol from the matrices. Fig. 7a illustrates for the example of $8 \%$ drug-loading how the release profiles for the matrices prepared from the purified Eudragit NE30D are much lower than those for the matrices prepared from the raw 

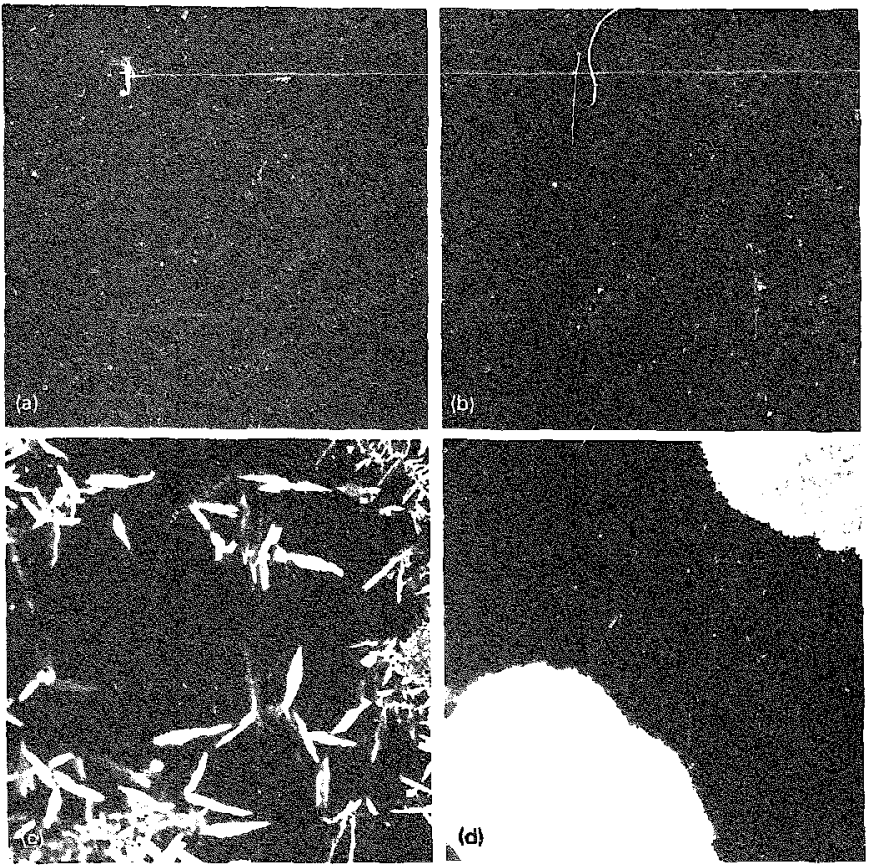

Fig. S. Photographs of 8-days' old matrices prepared from purified Eudragit NE30D viewed under crossed polarisers; (a) drugfree, (b) $6 \% \mathrm{w} / \mathrm{w}$ drug (clenbuterol) loading, (c) $12 \%$ drug loading, (d) $20 \%$ drug loading.

Eudragit NE30D (latter taken from ref. 2). The diffusivities calculated from the individual profiles are concentration-dependent for both raw and purified Eudragit NE30D-matrices (Fig. 7b). Increasing diffusivity with greater drug loading has already been observed for the nonextracted polymer $[1,2]$. The subsequent decrease observed here at high drug loading is readily explained by the influence of the presence of substantial amounts of suspended drug within the poiymer. For the calculation of diffusivity from the release profile, the concentration of drug in the matrix must be specified [2], all of which is assumed to exist in the dissolved state. In the presence of suspended drug, this value will be overestimated and cause an underestimation in the calculated diffusivity.

Fig. $7 \mathrm{~b}$ also illustrates that the diffusivities for the purified Eudragit NE30D-matrices are about $1 / 3$ of the magnitude of those for the raw Eudragit NE30D-matrices (latter also taken from ref. 2 ). The degree of scatter in the diffusivities ob- 
(a)

$\mathbf{E}$

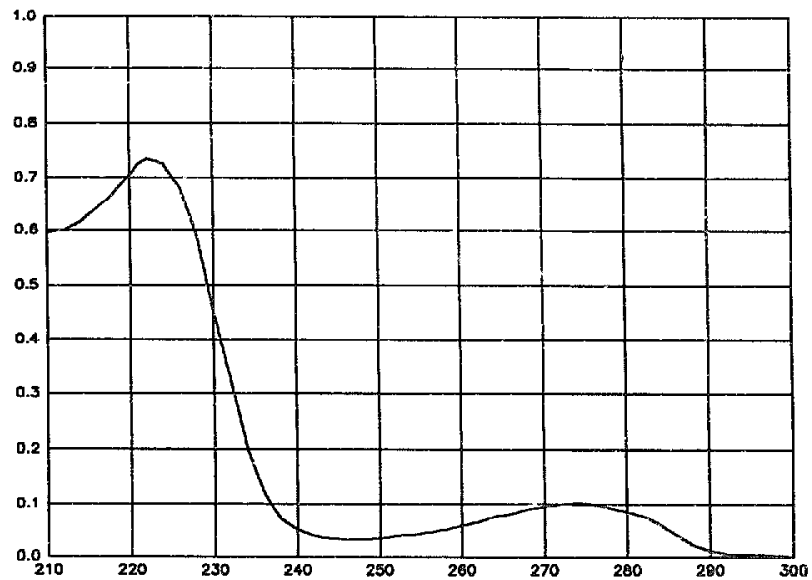

Wavelength [nm]

(b)

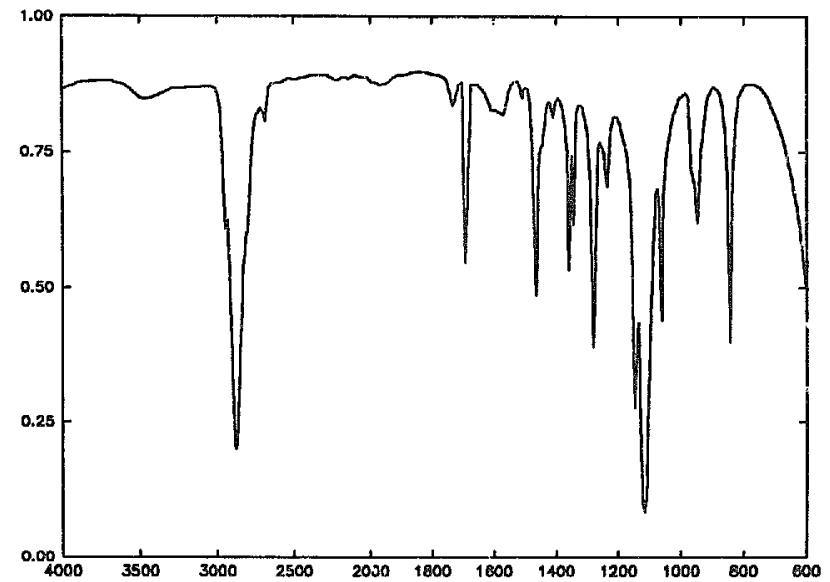

Wavenumber $\left[\mathrm{cm}^{-1}\right]$ 


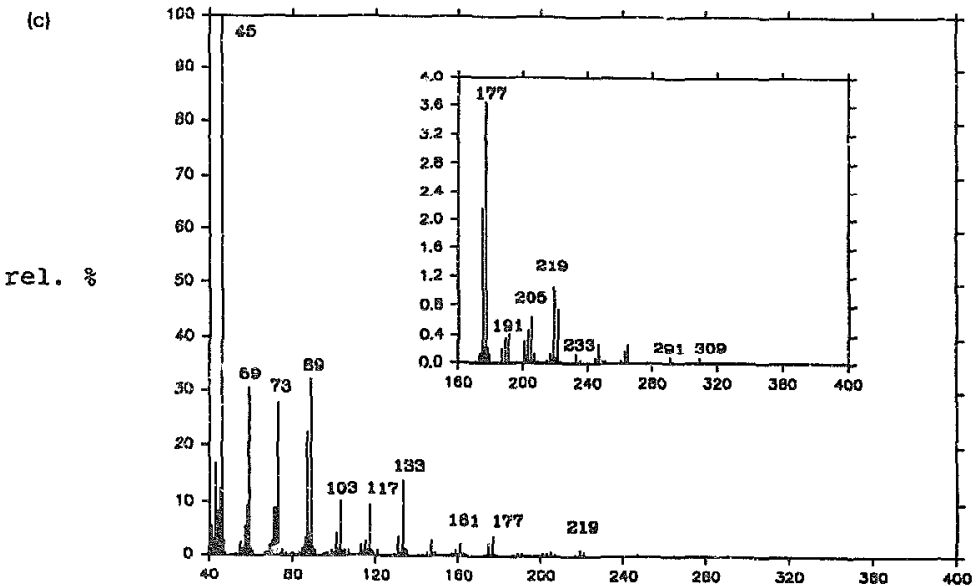

(d)

m/e

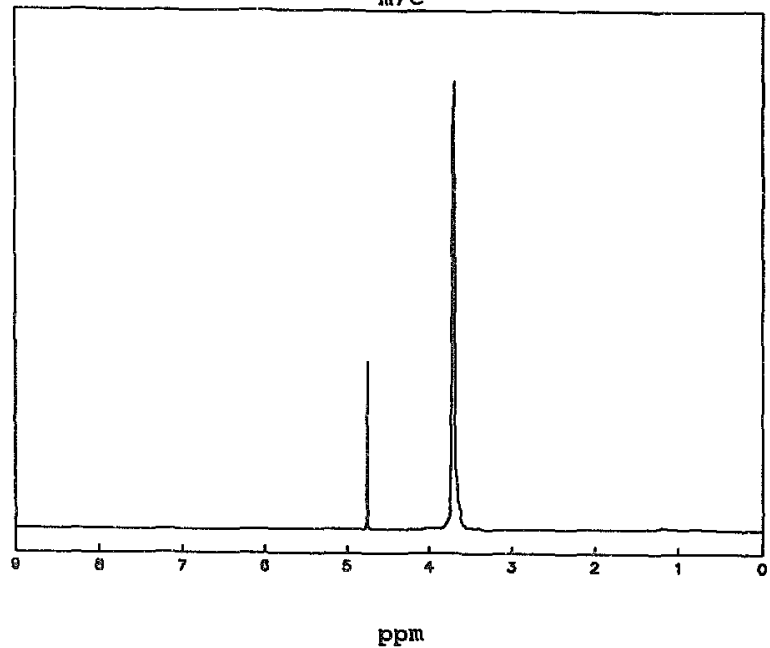

Fig. 6. Spectra obtained from the extract: (a) UV-spcetrum, (b) IR-spcctrum, \{cl mass-spectrum, (d) NMR-spectrum. 

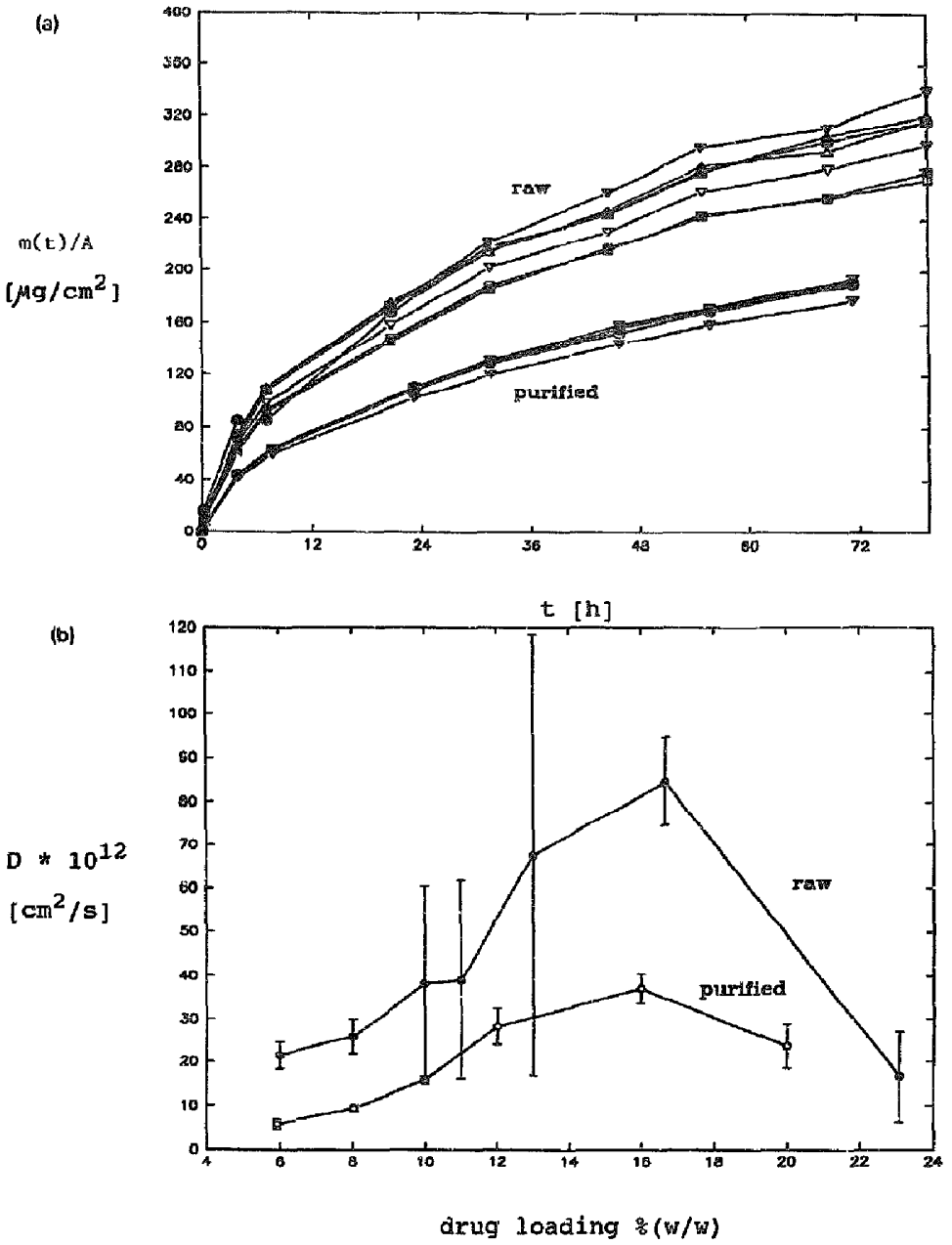

Fig. 7. (a) Drag release profiles for clenbuterol from raw and parified Eudragit NE30D matrices with $3 \%$ w/w drug ioading; (b) calculated diffusivities for clenbuterol in raw and puritied Eudragit NE30D-malrices as a function of drug loading (matrix thickness $=50 \mathrm{am}$ ). 
tained is also of note, being strongly dependent on drug loading. At the lowest drug loading of the raw Eudragit NE30D we find a coefficient of variation ( $\mathrm{CV}$ ) for the diffusivity of approx. $10 \%$, which increases sharply with greater daug loading. After reaching some $75 \%$ for $13 \%$ drug loading, the $\mathrm{CV}$ then falls to approx. $23 \%$ at the lighest drug loading examined. The CVs for the diffusivities for the purified Eudragit NE30D. matrices do not show this same dependence on drug loading. They are fairly constant in value, never being more than approx. 10-15\% for any of the drug loadings examined. The more scattered relcase profiles and diffusivities obtained for the raw Eudragit NE30D-matrices are perhaps not surprising considering the evident uncontrolled nature of the phase separation of the contaminant within the polymer. Assuming that this contaminant is the stated surfaciant, then the mechanism by which its presence leads to greater drug release rates may be related to improved wetting of the matrix surface or greater water uptake into the matrix. It is, however, of note that the presence of such a small quantity (approx. 3\%) of this endogenous contaminant has such a strong influence on the ratc of drug release.

\section{Conclusions}

Matrices prepared from thin films of Eudragit NE30D are anisotropic systems due to the presence of a ohace separated, water-soluble contaminant that is incompatible with the polymer. This is in all probability an o-substituted nonyl phenol surfactant used during the manufacture of the polymer. The removal of this surfactant by a simple agueous extraction process yields an isotropic material. Although the rate of drug release (clenbuterol) is thereby reduced by almost two thirds in magnitude, the degree of scatter in both the release profiles and calculated diffusivities within the matrix is substantially improved.

\section{Acknowledgement}

We thank Boehringer Ingelheim KG for its generous financial support of this project.

\section{References}

I B. Zierenberg, Einsatz des Nelder-Mead-Verfahrens zur Optimierung der Freigabcparameler yon Polymersystemen für Arzneistoffe, Acta Pharm. Technol., 30 (1985) $17-21$.

2 A. Göpferich and G. Lee, Measurement of drug diffusivities in stratum corneum membranes and a polyacrylate matrix, Int. J. Pharm., 7I (1991) 245-253.

3 L. Fan and S. Singh. Controlled Release: A Quantitative Treatment, Springer Verlag, Heidelberg, pp. 61-79, 1989.

4 K. Lehmann and $H$. Petereit, Use of aqueous Poly (meth \}acrylatc-dispersions for the manufacture of matrix tablets, Acta Pharm. Technol. 34 (1988) 189-195.

5 A. Schäffler, B. Zierenberg and H. Ludwig, Physicochemical characterisation and drug release of a matrix system containing polyacrylate and PVP. Acti Pharm. Technol., 36 (1990) $24 \mathrm{~S}$.

6 E. Nürnberg and J. Pfeulfer, Preparation and properties of a neutral polymethacrylate copolymer in powdercd form. Pharm. Ztg.-Wissensch.. 134 (1989) 294-299.

7 K. Lehmann, Chemistry and application properties of polymethacrylate coating systems, in: J. McGinity (Ed.), Aqueous Polymeric Cuatings for Pharmaceutical Dosage Forms, Marcel Dekker, New York, 1989; a) pp, 9-12; b) pp. $45-4 \dot{0}, 48$. 